\title{
Top reconstruction and boosted top: experimental overview
}

Louise Skinnari*, on behalf of the ATLAS and CMS Collaborations

Cornell University (US)

E-mail: louise.skinnariecern.ch

An overview of techniques used to reconstruct resolved and boosted top quarks is presented. Techniques for resolved top quark reconstruction include kinematic likelihood fitters and pseudotop reconstruction. Many tools and methods are available for the reconstruction of boosted top quarks, such as jet grooming techniques, jet substructure variables, and dedicated top taggers. Different techniques as used by ATLAS and CMS analyses are described and the performance of different variables and top taggers are shown.

8th International Workshop on Top Quark Physics, TOP2015

14-18 September, 2015

Ischia, Italy

\footnotetext{
* Speaker.
} 


\section{Introduction}

Reconstructing top quarks and top-quark pairs $(t \bar{t})$ involves identifying and correctly assigning observed decay products to the original top quark(s). Resolved top decays typically result in wellseparated jets and isolated leptons, while for boosted tops, the decay products may be overlapping, resulting in merged jets and non-isolated leptons. The separation of the decay products in $\eta-\phi$ can be approximated as $\Delta R \approx 2 m / p_{\mathrm{T}}$, where $\Delta R=\sqrt{\Delta \eta^{2}+\Delta \phi^{2}}, m$ the mass of the decaying particle, and $p_{\mathrm{T}}$ its transverse momentum. This corresponds to that a top quark with e.g. $p_{\mathrm{T}}=350 \mathrm{GeV}$ has its decay products contained in a cone of $\Delta R \approx 1.0$. These proceedings discuss different techniques used by ATLAS [1] and CMS [2] analyses.

\section{Resolved top reconstruction}

\subsection{Kinematic likelihood techniques}

Many techniques have been developed to associate the final state decay products (jets) to the partons from the original top quark. One technique for reconstructing resolved top quarks in $t \bar{t} \rightarrow$ $l+$ jets final states is a kinematic likelihood fitter, KLFitter [3], which uses a 3D template fit. The method aims to correctly assign jets to partons by maximizing a likelihood, testing each possible permutation of jet-parton association. The likelihood is formed using Breit-Wigner functions which constrain the reconstructed dijet/trijet mass to the mass of the $W$ boson $\left(m_{W}\right)$ or top quark $\left(m_{\text {top }}\right)$, and transfer functions which map the measured jet energy to the energy of the final state partons. The method is used in numerous ATLAS analyses such as the $7 \mathrm{TeV}$ top mass measurement [4], where the observables are the reconstructed top quark mass $\left(m_{\text {top }}^{\text {reco }}\right)$, the reconstructed $W$ mass $\left(m_{W}^{\text {reco }}\right)$, and a variable sensitive to the relative jet energy scale for $b$-quark jets vs light-quark jets.

The kinematic likelihood technique is also used by CMS for the $13 \mathrm{TeV}$ differential $t \bar{t}$ cross section measurement in the $l+$ jets channel [5]. The neutrino momentum is first reconstructed using $m_{W}$ and $m_{\text {top }}$ constraints. Using the solution best compatible with the measured missing transverse energy (MET) leads to an improved neutrino $p_{\mathrm{T}}$ measurement. Next, a likelihood for the most probable quark-to-jet assignment is defined from the $2 \mathrm{D}$ probability of the invariant masses of the 2-jets and 3-jets systems tested as the $W$ boson and hadronic top quark decay products, respectively, and the probability of the neutrino reconstruction to correctly select the $b$-jet. The combined reconstruction efficiency is about $60 \%$.

\subsection{Pseudo-top reconstruction}

Pseudo-top reconstruction aims to minimize the model dependence of differential cross-section measurements, allowing QCD precision tests in the top quark sector. The "pseudo top" is a topquark proxy object at stable-particle level. For $l+$ jets events, the leptonic $W$ is defined from the electron/muon and the MET, solving for the $z$-component of the neutrino momentum assuming $m_{W}$. The leptonic pseudo-top is formed from the leptonic $W$ and the closest $b$-jet. The hadronic $W$ is formed from the next two highest- $p_{\mathrm{T}}$ jets and the hadronic pseudo-top from this $W$ combined with the remaining $b$-jet. The usage of pseudo-top reconstruction avoids performing the largely model-dependent extrapolation from detector-level to parton-level $[6,7]$. 


\section{Boosted top reconstruction}

Boosted (high- $p_{\mathrm{T}}$ ) top quarks require dedicated reconstruction techniques due to their collimated decay products. Techniques for reconstructing boosted objects are used both to allow testing predictions of high- $p_{\mathrm{T}}$ top-quark production within the Standard Model as well as to probe models of physics beyond the Standard Model which result in boosted objects, such as new heavy resonances or vector-like quarks.

\subsection{Boosted leptonic tops}

For boosted top quarks which decay leptonically, standard isolation variables are suboptimal as the lepton and $b$-jet are typically not well-separated. Run-I ATLAS analyses used a shrinking isolation cone variable, defined by summing the $p_{\mathrm{T}}$ of tracks in a cone around the lepton with radius inversely proportional to the lepton $p_{\mathrm{T}}$. CMS instead relied on kinematic cuts, using a $2 \mathrm{D}$ variable requiring the lepton to either be separated in $\Delta R$ from the closest jet or have a component of $p_{\mathrm{T}}$ transverse to the axis of the closest jet.

\subsection{Boosted hadronic tops}

Boosted top quarks decaying hadronically are reconstructed by clustering the decay products into a single large-radius $(R)$ jet. Jet grooming techniques are applied to reduce contamination from pileup (PU), initial-state radiation (ISR), and underlying event (UE) to improve the separation in jet mass for signal vs background processes. In addition to the groomed jet mass, both jet substructure variables and dedicated top taggers are used to reconstruct boosted hadronic tops.

Jet grooming: Jet trimming [8], common in Run-I ATLAS analyses, reclusters jets into subjets, discarding subjets with small $p_{\mathrm{T}}$ (typically $\sim 5 \%$ of the original jet $p_{\mathrm{T}}$ ). Jet pruning [9], used in Run-I CMS analyses with boosted $W$ bosons, removes soft and wide-angle radiation as part of the jet algorithm. A new technique, soft drop [10], also removes wide-angle soft radiation through recursively declustering the jet. Soft drop is becoming the default in Run-2 for CMS as a powerful method in pushing the QCD jet mass to low values while preserving the hard scale of top quarks or heavy resonances. The soft drop mass for simulated $t \bar{t}$ and QCD multijet events is shown in Figure 1.

HEP Top Tagger: The HEP Top Tagger [11] starts from $R=1.5$ Cambridge-Aachen jets and declusters the jet into hard subjets using a "mass drop" criteria. All combinations of three subjets are tested for compatibility with a hadronic top decay. PU and UE contributions are removed by reclustering and filtering. Finally, the combination with filtered jet mass closest to the top mass is chosen. Kinematic cuts are applied to differentiate top quarks from background jets, requiring one subjet pair to have an invariant mass around $m_{W}$ and the combined jet to have a mass around $m_{\text {top }}$. The HEP Top Tagger was used by both ATLAS and CMS for moderately boosted top quarks $\left(p_{\mathrm{T}}>200 \mathrm{GeV}\right)$.

CMS Top Tagger: The CMS Top Tagger algorithm [12], used extensively in CMS Run-I analyses, declusters jets into up to four subjets, removing soft and wide-angle clusters. $R=0.8$ jets are used, resulting in an efficient reconstruction for $p_{\mathrm{T}}>400 \mathrm{GeV}$. Three subjets, minimum pairwise mass $>50 \mathrm{GeV}$, and a jet mass within an $m_{\text {top }}$ window are required. 


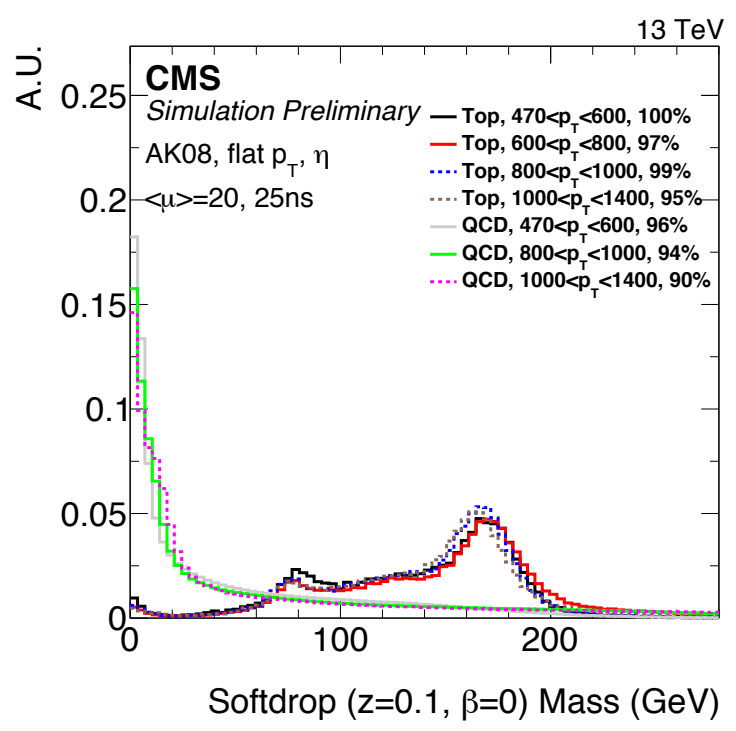

Figure 1: Distribution of soft drop mass for anti- $k_{t} R=0.8$ jets for different $p_{\mathrm{T}}$ ranges, comparing simulated $t \bar{t}$ and QCD multijet events at $13 \mathrm{TeV}$ from CMS [15].

Shower deconstruction: Shower deconstruction [13] defines a likelihood that a large- $R$ jet is due to a signal or background process. Using as input $R=0.1-0.2$ subjets, it is assumed that each subjet comes from a certain source of radiation such as a top decay or ISR. The probability that a given subjet configuration originates from a particular decay chain is then calculated. Shower deconstruction allows for a very pure signal selection.

$\mathrm{N}$-subjettiness: $\mathrm{N}$-subjettiness [14] is a jet shape variable used to measure the consistency of a jet to have $\mathrm{N}$ subjets. It is defined as the $p_{\mathrm{T}}$-weighted sum of distances of the constituent particles from the subjet axes: $\tau_{3} / \tau_{2}$ discriminates between a three-prong subjet structure (top) vs a non-top jet. This variable is shown in Figure 2(a).

$k_{t}$ splitting scale: Several Run-I ATLAS analyses use the $k_{t}$ splitting scale, a measure of the scale of the last recombination step in the $k_{t}$ jet clustering algorithm which clusters high- $p_{\mathrm{T}}$ and large-angle components last. The observable corresponding to the splitting scale of the last step, $\sqrt{d_{12}}$, is shown in Figure 2(b). For large- $R$ jets in $t \bar{t}$ signal, it peaks around $m_{\text {top }} / 2$.

Subjet $b$-tagging: Since top decays involve a $b$-quark, applying subjet $b$-tagging can significantly increase the QCD multijet background rejection. ATLAS defines an improved $b$-tagger for additional discrimination in the boosted regime (MVb) [17] and CMS uses an improved tagger which uses as input an inclusive vertex finder algorithm independent, by construction, of the jet algorithm used [18].

The performance of different top taggers and jet substructure variables are studied using simulation. Figure 3(a) shows the background rejection as a function of the tagging efficiency at $8 \mathrm{TeV}$ for ATLAS. The best performance in terms of large background rejection is achieved for shower deconstruction. A common working point in Run-I included trimmed jet mass $>100 \mathrm{GeV}$ with cuts on the $k_{t}$ splitting scale. Figures 3(b) and 3(c) show similar performance comparisons from CMS at $13 \mathrm{TeV}$. Shower deconstruction is again the single most discriminating variable, though 


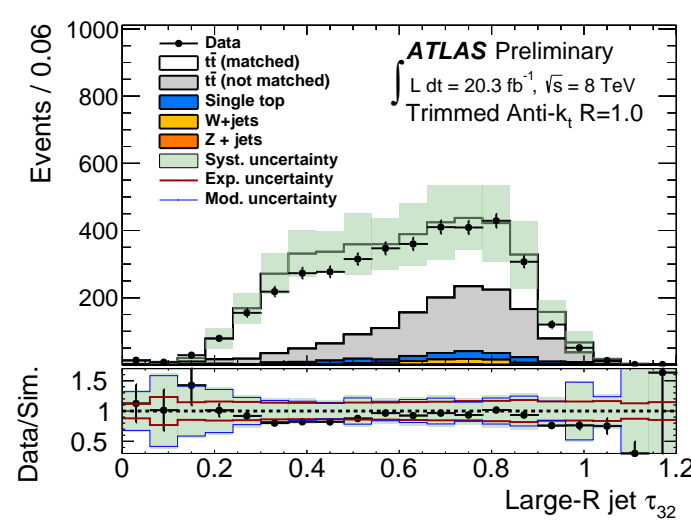

(a)

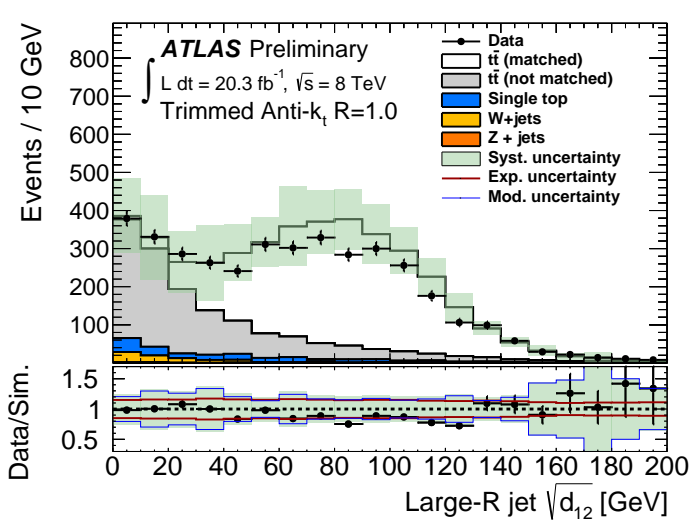

(b)

Figure 2: Distribution of (a) the N-subjettiness ratio $\tau_{3} / \tau_{2}$ and (b) the splitting scale $\sqrt{d_{12}}$ for trimmed $R=1.0$ jets in events passing a $t \bar{t}$ signal selection at $8 \mathrm{TeV}$ from ATLAS [16].

it does not reach as high efficiency. For combined taggers, similar performance is achieved for a variety of variables.

\section{Conclusions}

In conclusion, many different methods are used for reconstructing resolved and boosted top quarks. Jet substructure techniques are used extensively in identifying boosted hadronic top quarks in searches for beyond the Standard Model physics and more recently also in top quark crosssection measurements targeting the high- $p_{\mathrm{T}}$ regime. With higher center-of-mass energy, boosted objects will become even more important in Run-II. 


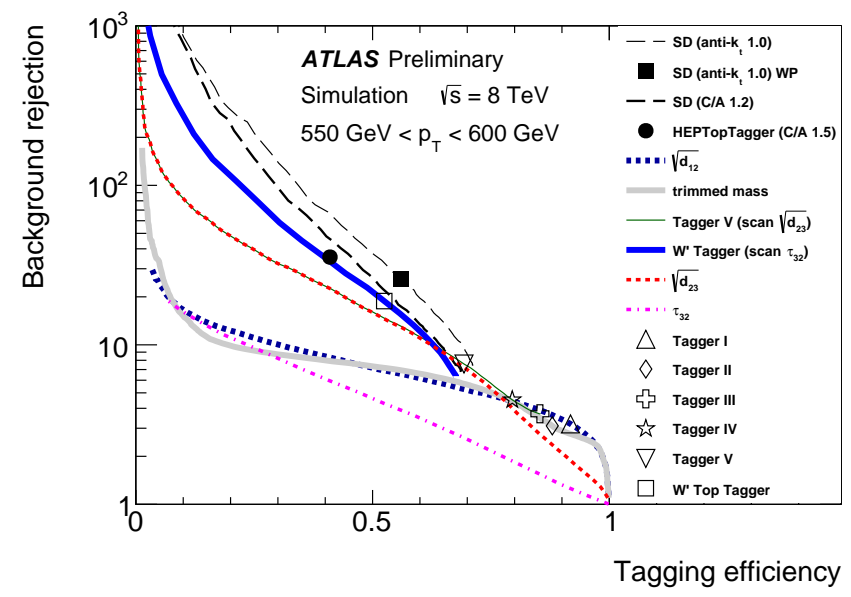

(a)
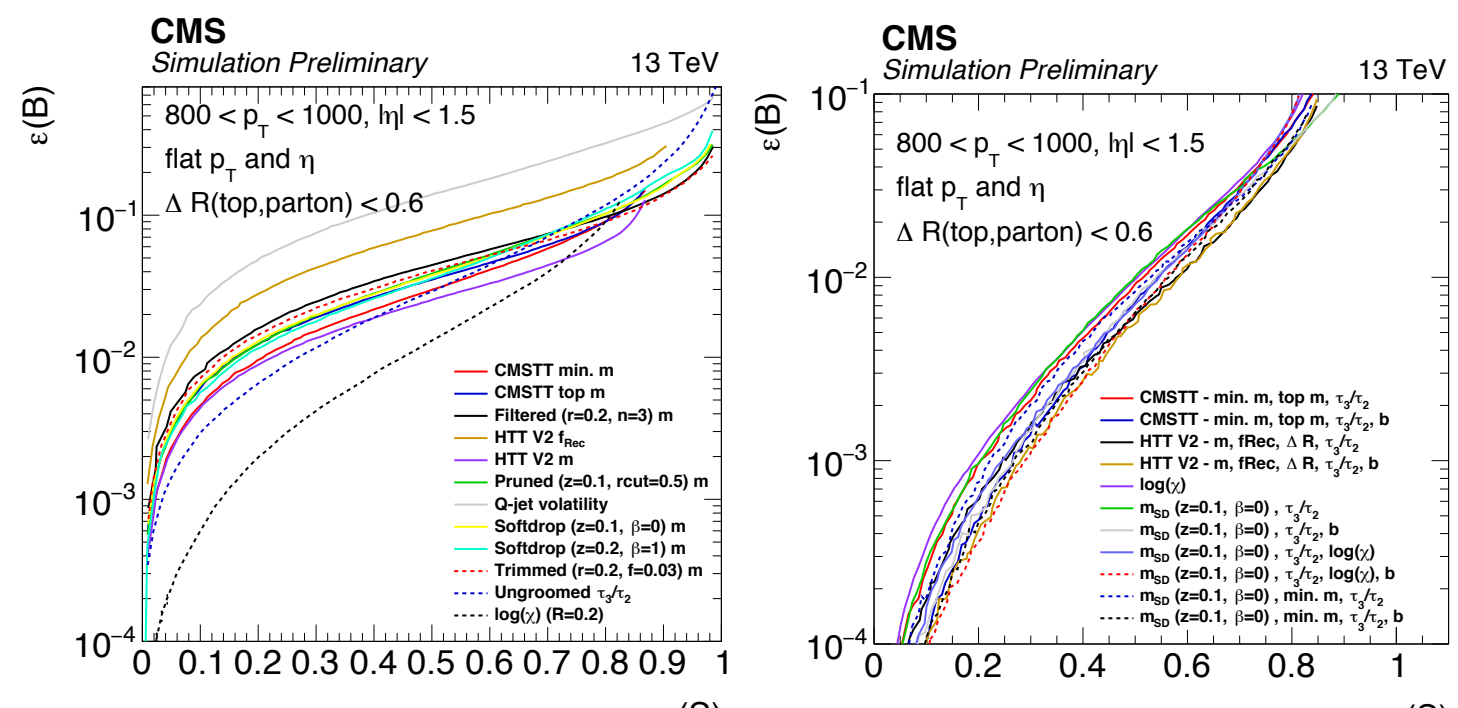

$\varepsilon(\mathrm{S})$

(b)

(c)

Figure 3: Background rejection as a function of tagging efficiency shown for large- $R$ jets with $550<p_{\mathrm{T}}<$ $600 \mathrm{GeV}$ for different top taggers from ATLAS at $8 \mathrm{TeV}$ in (a) [16]. Taggers I-V correspond to different working points using $\mathrm{N}$-subjettiness, $k_{t}$ splitting scale, and trimmed jet mass. Background efficiency as a function of tagging efficiency shown for large- $R$ jets with $800<p_{\mathrm{T}}<1000 \mathrm{GeV}$ for (b) single variables and (c) combined variables at $13 \mathrm{TeV}$ from CMS [15]. 


\section{References}

[1] ATLAS Collaboration, The ATLAS Experiment at the CERN Large Hadron Collider, JINST 3 (2008) S08003.

[2] CMS Collaboration, The CMS Experiment at the CERN LHC, JINST 3 (2008) S08004.

[3] J. Erdmann et al, A likelihood-based reconstruction algorithm for top-quark pairs and the KLFitter framework, Nucl. Instrum. Meth. A748 (2014).

[4] ATLAS Collaboration, Measurement of the top quark mass in the $t \bar{t} \rightarrow$ lepton $+j e t s$ and $t \bar{t} \rightarrow$ dilepton+jets channels using $\sqrt{s}=7$ TeV ATLAS data, Eur. Phys. J. C (2015) 75:330.

[5] CMS Collaboration, Measurement of the inclusive and differential top quark pair production cross sections in lepton+jets final states at $\sqrt{s}=13 \mathrm{TeV}$, CMS-PAS-TOP-15-005 (2015).

[6] ATLAS Collaboration, Differential top-antitop cross-section measurements as a function of observables constructed from final-state particles using pp collisions at $\sqrt{s}=7 \mathrm{TeV}$ in the ATLAS detector, JHEP 06 (2015) 100.

[7] CMS Collaboration, Measurement of the differential cross section for top quark pair production in pp collisions at $\sqrt{s}=8 \mathrm{TeV}$, Eur. Phys. J. C 75 (2015) 542.

[8] D. Krohn, J. Thaler, L. T. Wang, Jet Trimming, JHEP 1002 (2010) 084.

[9] S. D. Ellis, C. K. Vermilion, J. R. Walsh, Recombination Algorithms and Jet Substructure: Pruning as a Tool for Heavy Particle Searches, Phys. Rev. D 81 (2010) 094023.

[10] A. J. Larkoski, S. Marzani, G. Soyez, J. Thaler, Soft Drop, JHEP 1405 (2014) 146.

[11] T. Plehn, M. Spannowsky, M. Takeuchi, D. Zerwas, Stop Reconstruction with Tagged Tops, JHEP 1010 (2010) 078.

[12] CMS Collaboration, Boosted top jet tagging at CMS, CMS-PAS-JME-13-007 (2014).

[13] D. E. Soper, M. Spannowsky, Finding top quarks with shower deconstruction, Phys. Rev. D 87 (2013) 054012.

[14] J. Thaler, K. Van Tilburg, Identifying Boosted Objects with N-subjettiness, JHEP 1103 (2011) 015.

[15] CMS Collaboration, W/Z/Top tagging for Run 2 in CMS, CMS DP-2015/043 (2015).

[16] ATLAS Collaboration, Identification of high transverse momentum top quarks in pp collisions at $\sqrt{s}=8 \mathrm{TeV}$ with the ATLAS detector, ATLAS-CONF-2015-036 (2015).

[17] ATLAS Collaboration, b-tagging in dense environments, ATL-PHYS-PUB-2014-014 (2014).

[18] CMS Collaboration, Performance of b-tagging in boosted topology events, CMS DP-2014/031 (2014). 